\title{
Proposal of Sporolactobacillus nakayamae subsp. nakayamae sp. nov., subsp. nov., Sporolactobacillus nakayamae subsp. racemicus subsp. nov., Sporolactobacillus terrae sp. nov., Sporolactobacillus kofuensis sp. nov., and Sporolactobacillus lactosus sp. nov.

\author{
FUJITOSHI YANAGIDA, ${ }^{1 *}$ KEN-ICHIRO SUZUKI, ${ }^{2}$ MICHIO KOZAKI, ${ }^{3}$ AND KAZUO KOMAGATA ${ }^{4}$ \\ The Institute of Enology and Viticulture, Yamanashi University, Kofu-shi, Yamanashi $400,{ }^{1}$ Japan Collection of \\ Microorganisms, The Institute of Physical and Chemical Research (RIKEN), Wako-shi, Saitama 351-01, ${ }^{2}$ Doctor \\ Course of Science for Living System, Showa Women's University, Setagaya-ku, Tokyo 154, ${ }^{3}$ and Department of \\ Agricultural Chemistry, Tokyo University of Agriculture, Setagaya-ku, Tokyo $156,{ }^{4}$ Japan
}

\begin{abstract}
Catalase-negative spore-forming lactic acid bacteria were isolated from soil samples and fermentation starters for Asian traditional alcoholic beverages. The isolates were characterized by determining morphological, biochemical, physiological, and chemotaxonomic properties and were found to be members of the genus Sporolactobacillus. Twelve isolates and some authentic strains belonging to this genus were used in DNA base composition and DNA relatedness studies, and the results revealed that the strains tested could be divided into six groups which correlated with the phenotypic characteristics. One of the groups corresponded to the previously described species Sporolactobacillus inulinus. In addition, we propose the following four new species and two new subspecies for the remaining five groups: Sporolactobacillus nakayamae subsp. nakayamae sp. nov., subsp. nov. (type strain, M-114 [= JCM 3514]), Sporolactobacillus nakayamae subsp. racemicus subsp. nov. (type strain, M-17 [= JCM 3417]), Sporolactobacillus terrae sp. nov. (type strain, M-116 [= JCM 3516]), Sporolactobacillus kofuensis sp. nov. (type strain, M-19 [= JCM 3419]), and Sporolactobacillus lactosus sp. nov. (type strain, X1-1 [= JCM 9690]).
\end{abstract}

Originally, the genus Sporolactobacillus consisted of catalase-negative, spore-forming, homofermentative lactic acid bacteria and contained a single species, Sporolactobacillus inulinus, which was first isolated from chicken feed $(13,14)$. Later, Nakayama and Yanoshi $(18,19)$ isolated a number of sporeforming lactic acid bacteria from the soil of plant rhizospheres and proposed four species for them. However, the names proposed by Nakayama and Yanoshi did not appear on the Approved Lists of Bacterial Names (24) and have not been validly published. Previously, we studied the strains isolated by $\mathrm{Na}$ kayama and Yanoshi $(18,19)$, as well as the type strains of $S$. inulinus, Lactobacillus plantarum, and Bacillus coagulans, by performing morphological, biochemical, and physiological characterization (32) and DNA-DNA hybridization (33) experiments. These studies revealed that the genus Sporolactobacillus is phenotypically distinct from the genus Bacillus; in particular, it lacks catalase activity and requires carbohydrates for growth. The strains of the genus Sporolactobacillus have been divided into several groups. Fox et al. (7) described a distant relationship between $S$. inulinus and Bacillus species based on $16 \mathrm{~S}$ rRNA oligonucleotide cataloging data. Ash et al. (2) classified the genus Bacillus into five groups by performing a comparative analysis of $16 \mathrm{~S}$ rRNA primary sequences (ca. $95 \%$ of the total molecule), and $S$. inulinus was phylogenetically separated from all of the Bacillus species by the results. Suzuki and Yamasato (27) determined the 16S rRNA gene sequences of 16 strains of spore-forming lactic acid bacteria and found that the isolates of Nakayama and Yanoshi formed one cluster with $S$. inulinus and that this cluster could be further divided into five subclusters, including four catalase-negative subclusters

\footnotetext{
* Corresponding author. Mailing address: The Institute of Enology and Viticulture, Yamanashi University, 1-13-1, Kitashin, Kofu-shi, Yamanashi, 400, Japan. Phone: 81-552-20-8605. Fax: 81-552-20-8606. Email: yanagida@yu-gate.yamanashi.ac.jp.
}

and one catalase-positive subcluster. Recently, Andersch et al (1) revived the name Bacillus laevolacticus for a group of catalase-positive strains. Although the genus Sporolactobacillus has been studied taxonomically frequently, species other than $S$. inulinus have not been established previously. As isolation sources of Sporolactobacillus strains were rather limited, we tried to isolate strains of this genus from fermentation starters of alcoholic beverages in Asian countries and from soil samples. In this paper we describe isolation of strains belonging to the genus Sporolactobacillus and propose four new species and two new subspecies, Sporolactobacillus nakayamae subsp. nakayamae, Sporolactobacillus nakayamae subsp. racemicus, Sporolactobacillus terrae, Sporolactobacillus kofuensis, and Sporolactobacillus lactosus, for our isolates and the strains isolated by Nakayama and Yanoshi $(18,19)$.

\section{MATERIALS AND METHODS}

Isolation procedure. Sixty soil samples were collected mainly from the rhizospheres of plants growing in wet regions in various parts of Japan. Twelve samples of fermentation starters of traditional alcoholic beverages in Asian countries, including luck pang in Thailand, bubod in the Philippines, ragi in Indonesia, and lepar hu in Bhutan, were also used as isolation sources. A 1-g portion of a sample was mixed with $5 \mathrm{ml}$ of GYP medium, which contained $2 \%$ glucose, $1 \%$ yeast extract, $1 \%$ peptone, $1 \%$ sodium acetate, and $0.5 \%(\mathrm{vol} / \mathrm{vol})$ salts solution (pH 6.8). The salts solution contained $4 \% \mathrm{MgSO}_{4} \cdot 7 \mathrm{H}_{2} \mathrm{O}, 0.2 \%$ $\mathrm{MnSO}_{4} \cdot 4 \mathrm{H}_{2} \mathrm{O}, 0.2 \% \mathrm{FeSO}_{4} \cdot 7 \mathrm{H}_{2} \mathrm{O}$, and $0.2 \% \mathrm{NaCl}$. Each sample suspension was heated at $80^{\circ} \mathrm{C}$ for $10 \mathrm{~min}$ and then incubated anaerobically at $30^{\circ} \mathrm{C}$ to remove non-spore-forming aerobic bacteria. After 4 to 7 days of incubation, drop of the turbid broth was spread onto GYP agar containing $1 \% \mathrm{CaCO}_{3}$, and the culture was incubated in an anaerobic jar in a $100 \% \mathrm{CO}_{2}$ atmosphere. Acid-producing bacteria were recognized by the appearance of clear zones around colonies. These bacteria were purified by repeated isolation, and the production of lactic acid was confirmed by high-performance liquid chromatography. A Shodex Ionpack KC-811 column was used with $0.1 \% \mathrm{H}_{3} \mathrm{PO}_{4}$ as the eluent $(1 \mathrm{ml} / \mathrm{min})$ at $60^{\circ} \mathrm{C}$, and lactic acid was detected by refractometry. Some of the strains, those lacking catalase activity, were used in further taxonomic studies. The catalase activity of cells grown on GYP agar slants (32) was determined by adding a $3 \%$ hydrogen peroxide solution. 
Strains used and cultivation. $S$. inulinus $\mathrm{JCM} 6014^{\mathrm{T}}(\mathrm{T}=$ type strain) was obtained from the Japan Collection of Microorganisms, The Institute of Physical and Chemical Research (RIKEN), Wako-shi, Saitama, Japan. The 30 strains whose designations begin with $\mathrm{M}$ - were strains isolated by Nakayama and Yanoshi $(18,19)$. The strains were cultivated on GYP medium at $30^{\circ} \mathrm{C}$ unless otherwise stated (32)

Morphological, biochemical, and physiological characteristics. Conventional tests were performed by using previously described methods (32). Acid production from carbohydrates was examined by the method of Mitsuoka (17), modified by using liquid media without $\mathrm{L}-\mathrm{cysteine}-\mathrm{HCl}$. The isomers of lactic acid produced were determined by the enzymatic method described by Okada et al. (21).

Chemotaxonomy. The presence of diaminopimelic acid isomers in cell walls was determined by the method of Staneck and Roberts (25) by using whole-cell hydrolysates. Cellular fatty acid compositions were analyzed by using methyl esters prepared from lyophilized cells with anhydrous $5 \%$ methanolic hydrochloric acid (Kokusan Chemical Works, Tokyo, Japan) as described by Ikemoto et al. (10). A model GC-7A gas chromatograph (Shimadzu Corp., Kyoto, Japan) equipped with a Rascot OV-1 capillary column $(50 \mathrm{~m}$ by $0.25 \mathrm{~mm}$; Nihon Chromato, Tokyo, Japan) was used to determine the composition. Isoprenoid quinones were extracted from lyophilized cells with chloroform-methanol (2:1, $\mathrm{vol} / \mathrm{vol}$ ) and were purified by thin-layer chromatography; the isoprenoid quinone composition was determined by reverse-phase high-performance liquid chromatography $(15,29)$.

DNA studies. Cells grown on GYP medium at $30^{\circ} \mathrm{C}$ were collected by centrifugation at the late exponential growth phase. DNA was isolated by the phenol method of Saito and Miura (22) with some modifications, as described previously (33). The DNA base composition was calculated from the melting temperature $\left(T_{m}\right)$ of DNA by using the formula of Marmur and Doty (16). The $T_{m}$ was determined with a model DU-8 spectrophotometer (Beckman Instruments, Inc. Fullerton, Calif.). The sodium dodecyl sulfate-membrane filter method described previously $(26,33)$ was used for DNA-DNA hybridization experiments.

\section{RESULTS}

Isolation of bacteria. Twenty strains of spore-forming lactic acid bacteria were isolated from soil samples, and 15 strains were isolated from fermentation starters. The proportions of catalase-negative strains among the spore-forming lactic acid bacteria isolated from soil samples and fermentation starters were 39 and $63 \%$, respectively. The strains with catalase activity were not included in this study. Twelve representative strains were selected for further study. Strains MB-1, ME-1', and $\mathrm{MH}-3$ were isolated from soil samples obtained in Yamanashi Prefecture. Strains F-4, I-3, and SH-3 were isolated from soil samples obtained in Saitama, Shizuoka, and Tokushima Prefectures, respectively. The strains isolated from Asian fermentation starters were strain $\mathrm{X} 1-1^{\mathrm{T}}$ from luck pang in Thailand, strain X3-1 from bubod in the Philippines, strains X5-5 and X6-1 from ragi in Indonesia, and strains $X 9-1$ and $X 9-3$ from lepar hu in Bhutan.

Morphological, biochemical, and physiological characteristics. All of the isolates were gram-positive rods and produced endospores that were resistant to heating at $80^{\circ} \mathrm{C}$ for $10 \mathrm{~min}$. Motility was observed with all of the isolates except strain $\mathrm{X} 1-1^{\mathrm{T}}$. Morphological, biochemical, and physiological characteristics of the isolates are shown in Table 1 along with the characteristics of strains described previously (32).

Chemotaxonomic characteristics. All of the isolates had meso-diaminopimelic acid in their whole-cell hydrolysates. The predominant isoprenoid quinone of the isolates was a menaquinone with seven isoprene units (MK-7); MK-5 and MK-6 were minor components. Ubiquinone was not detected. The major components of the cellular fatty acids of the isolates were 12-methyl tetradecanoic acid (anteiso- $\mathrm{C}_{15: 0}$ ) and 14methyl hexadecanoic acid (anteiso- $\mathrm{C}_{17: 0}$ ); 13-methyl tetradecanoic acid (iso- $\mathrm{C}_{15: 0}$ ) and 14-methyl pentadecanoic acid (iso$\mathrm{C}_{16: 0}$ ) were also present.

DNA base compositions and DNA relatedness. DNA base compositions and levels of DNA relatedness are shown in Table 2. The DNA base compositions of the isolates ranged from 43 to $47 \mathrm{~mol} \% \mathrm{G}+\mathrm{C}$. Strains X3-1, X5-5, X6-1, X9-1,
X9-3, SH-3, MB-1, MH-3, and I-3 exhibited high levels of DNA relatedness to strain $X 1-1^{\mathrm{T}}$ and formed a DNA homology group. Although strains ME-1' and F-4 exhibited low levels of relatedness to strain $\mathrm{X} 1-1^{\mathrm{T}}$, these isolates were closely related to strain $\mathrm{M}-114^{\mathrm{T}}$, one of the isolates of Nakayama and Yanoshi (19). None of the isolates exhibited a high level of DNA relatedness to the type strain of $S$. inulinus.

\section{DISCUSSION}

Catalase-negative spore-forming lactic acid bacteria were isolated from some fermentation starters and soil samples. All of the isolates contained meso-diaminopimelic acid in their cell walls, MK-7 was the predominant isoprenoid quinone, and the cellular fatty acids were anteiso- and iso-branched-chain fatty acids. The DNA base compositions ranged from 43 to 50 mol\% $\mathrm{G}+\mathrm{C}$. These chemotaxonomic properties were consistent with previous descriptions of the genus Sporolactobacillus $(5,12-14,30,31)$. The salient characteristics of the six genera of aerobic, endospore-forming rods and cocci are shown in Table 3 .

As in the previous studies of Sporolactobacillus strains $(6,14$ 19 ), a wide distribution of this genus was found in this study. Furthermore, species diversity was also found in the genus Sporolactobacillus. The isolates of Nakayama and Yanoshi (18, 19) were classified into five groups based on DNA relatedness, as shown previously (33). It is interesting that most of our isolates used in the present study did not belong to any of these five groups; the only exceptions were strains ME-1' and F-4, which fell into group 2 . The remaining isolates aggregated in a sixth group (Table 2). The six groups based on DNA relatedness data were well-characterized phenotypically and were consistent with the biochemical and physiological properties, as shown in Table 1 . Group 1 contains $S$. inulinus. Strain M-86 exhibited a high level of DNA relatedness to the type strain of $S$. inulinus, strain JCM 6014, and most of the phenotypic characteristics of these two strains were similar (the only exceptions were motility, growth at $45^{\circ} \mathrm{C}$, and acid production from sorbitol and inulin). The strains belonging to the five other groups are distinct from $S$. inulinus and can be classified in five new taxa based on phenotypic characteristics and DNA relatedness data. However, the levels of DNA relatedness between group 2 and group 4 are slightly higher than the levels of relatedness among the other groups and are within the range of levels of relatedness for different subspecies of the same species, as proposed by Johnson (11). Therefore, below we describe two new subspecies of one new species for the strains belonging to groups 2 and 4. We also describe three new Sporolactobacillus species for groups 3, 5, and 6. The taxa of the genus Sporolactobacillus, including $S$. inulinus, are distinguished from one another phenotypically by a combination of characteristics (Table 4).

Ash et al. (2) performed a phylogenetic analysis based on 16S ribosomal DNA sequences and found a great deal of diversity in the genus Bacillus. S. inulinus was placed on an independent branch within the large Bacillus group. Furthermore, Suzuki and Yamasato (27) found that 12 representative strains of spore-forming lactic acid bacteria isolated by $\mathrm{Na}$ kayama and Yanoshi $(18,19)$ and $S$. inulinus formed one cluster with very similar $16 \mathrm{~S}$ rDNA sequences (levels of similarity, more than $97.2 \%$ ). In addition, these authors mentioned that this cluster contains some catalase-positive strains and that the definition of the genus Sporolactobacillus should be amended to accommodate both catalase-negative and catalase-positive strains (27). Apart from this problem, it is evident that the 
TABLE 1. Biochemical and physiological characteristics of spore-forming lactic acid bacteria

\begin{tabular}{|c|c|c|c|c|c|c|c|c|c|c|c|c|c|c|c|c|c|c|c|c|c|c|c|c|c|c|c|c|c|c|c|c|c|c|c|c|}
\hline \multirow[b]{2}{*}{ 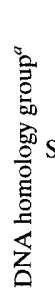 } & \multirow[b]{2}{*}{ Strain } & \multirow[b]{2}{*}{ 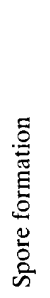 } & \multirow{2}{*}{\multicolumn{2}{|c|}{ 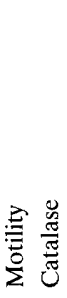 }} & \multicolumn{5}{|c|}{ Growth at: } & \multicolumn{5}{|c|}{$\begin{array}{l}\text { Growth in the } \\
\text { presence of: }\end{array}$} & \multicolumn{4}{|c|}{ Litmus milk } & \multicolumn{18}{|c|}{ Acid production from carbohydrates } \\
\hline & & & & & & & & & & $\begin{array}{l}\bar{U} \\
\bar{Z} \\
\text { so } \\
m\end{array}$ & $\begin{array}{l}\underbrace{}_{\pi} \\
Z \\
s^{\circ} \\
\dot{\sigma}\end{array}$ & $\begin{array}{l}\bar{J} \\
\ddot{z} \\
i \\
i\end{array}$ & $\begin{array}{l}U_{\pi} \\
Z \\
20 \\
i\end{array}$ & $\begin{array}{l}\vec{U} \\
Z \\
z \\
80 \\
\stackrel{0}{0}\end{array}$ & 莺 & 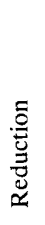 & 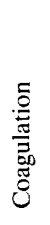 & 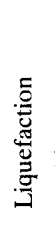 & 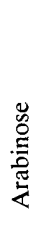 & 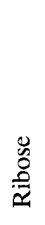 & $\frac{\mathscr{E}}{\grave{x}}$ & 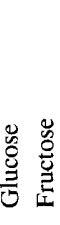 & 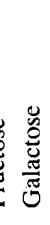 & $\frac{\mathscr{J}}{\mathscr{E}}$ & 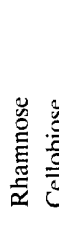 & 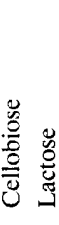 & 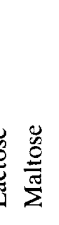 & 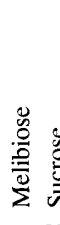 & 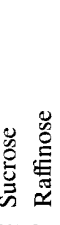 & 恶 & 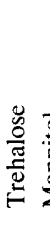 & 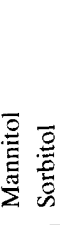 & $\frac{5}{0}$ & 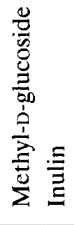 & $\stackrel{0}{\stackrel{\Xi}{\Xi}}$ & 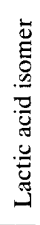 \\
\hline 1 & $\mathrm{JCM} 6014^{\mathrm{T} b}$ & $t^{d}$ & + & - & -1 & ++ & & - & - & + & + & + & + & - & - & - & - & - & - & - & - & ++ & - & + & - & - & -+ & & ++ & - & +- & ++ & + & ++ & - & $\mathrm{D}$ \\
\hline & $M-86^{c}$ & + & - & - & - & ++ & + & + & - & + & + & + & + & - & - & - & - & - & - & - & - & ++ & - & + & - & -- & -+ & -+ & ++ & - & + & +- & -+ & +- & - & $\mathrm{D}$ \\
\hline 2 & M-45 & + & + & - & + & ++ & + & - & - & + & + & + & + & - & - & - & - & - & - & - & - & ++ & + & + & - & -- & -+ & ++ & +- & - & + & - & - & - & - & D \\
\hline & M-46 & + & + & - & + & ++ & + & - & - & + & + & + & + & - & - & - & - & - & - & - & - & ++ & -+ & + & - & -- & -+ & -+ & $+\quad-$ & - & +- & -- & - & -- & - & D \\
\hline & M-47 & + & + & - & + & ++ & + & - & - & + & + & + & + & - & - & - & - & - & - & - & - & ++ & + & + & -+ & +- & -+ & ++ & +- & + & + & -- & -- & +- & - & D \\
\hline & M-50 & + & + & - & + & ++ & + & - & - & + & + & + & + & - & - & - & - & - & - & - & - & ++ & + & + & - & -- & -+ & -+ & +- & - & + & -- & -- & -- & - & $\mathrm{D}$ \\
\hline & M-51 & + & + & - & + & $+\quad+$ & + & - & - & + & + & + & + & - & + & - & - & - & - & - & - & ++ & + & + & - & -- & -+ & -+ & ++ & - & + & -+ & + & +- & - & $\mathrm{D}$ \\
\hline & M-54 & + & + & - & + & ++ & + & - & - & + & + & + & + & - & + & - & - & - & - & - & - & ++ & + & + & - & - & -+ & -+ & $+\quad-$ & - & + & - & -- & -- & - & D \\
\hline & M-5 & + & + & - & + & ++ & + & - & - & + & + & + & + & - & - & 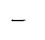 & 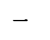 & - & - & - & - & ++ & -+ & + & - & -- & -+ & -+ & +- & - & + & +- & -- & +- & - & D \\
\hline & M-59 & + & + & - & + & ++ & + & - & - & + & + & + & + & - & + & - & - & - & - & - & - & ++ & + & + & - & -- & -+ & - & -- & - & + & -- & -- & -- & - & D \\
\hline & M-84 & + & + & - & + & ++ & + & - & - & + & + & + & - & - & + & - & - & - & - & - & - & ++ & -+ & + & - & -- & -+ & -+ & +- & - & + & +- & -- & -+ & - & D \\
\hline & M-87 & + & + & - & - & ++ & + & + & - & + & + & + & + & - & - & - & - & - & - & - & - & ++ & - & + & - & - & -+ & -+ & ++ & - & + & ++ & +- & +- & - & D \\
\hline & M-8 & + & + & - & - & ++ & + & - & - & + & + & + & - & - & - & - & - & - & - & - & - & ++ & -+ & + & - & -- & -+ & -+ & $+\quad+$ & - & + & ++ & $t+$ & +- & - & $\mathrm{D}$ \\
\hline & M-1 & + & + & - & + & ++ & + & - & - & + & + & + & - & - & - & - & - & - & - & - & - & ++ & + & + & - & -- & -+ & -+ & ++ & - & + & ++ & - & +- & - & D \\
\hline & M-10 & + & + & - & + & ++ & + & - & - & + & + & + & - & - & - & - & - & - & - & - & - & ++ & + & + & - & $-\quad-$ & -+ & -+ & ++ & - & + & -- & -- & ++ & - & D \\
\hline & M-11 & - & + & - & $+t$ & ++ & + & - & - & + & + & - & - & - & - & + & - & - & - & - & - & ++ & + & + & - & -- & -+ & -+ & $+\quad-$ & - & + & -- & -- & -- & - & D \\
\hline & $M-114^{T}$ & + & + & - & $+t$ & ++ & + & - & - & + & + & + & + & - & - & - & - & - & - & - & - & ++ & -+ & + & - & $-\quad-$ & -+ & -+ & ++ & - & - & ++ & + & - & - & D \\
\hline & $\mathrm{ME}$ & + & + & - & + & ++ & + & - & - & + & + & - & - & - & - & - & - & - & - & - & - & ++ & + & + & - & $-\quad-$ & -+ & -+ & ++ & - & + & ++ & +- & -- & - & D \\
\hline & F-4 & + & + & - & + & ++ & + & + & - & + & + & + & + & - & - & + & - & - & - & - & - & ++ & + & + & - & -- & -+ & -+ & ++ & - & +- & -+ & - & +- & - & $\mathrm{D}$ \\
\hline 3 & $M$ & + & + & - & + & ++ & + & - & - & + & + & - & - & - & - & - & - & - & - & - & - & ++ & + & + & - & - & -+ & -+ & +- & + & + & -- & -- & ++ & - & D \\
\hline & M-3 & + & + & - & + & $+\quad+$ & + & - & - & + & + & - & - & - & - & - & - & - & - & - & - & ++ & + & + & - & -- & -+ & - & -+ & - & + & +- & + & ++ & - & D \\
\hline & M-33 & - & + & - & + & ++ & + & - & - & + & + & - & - & - & - & - & . & - & - & - & - & ++ & - & + & - & -- & -+ & -+ & +- & - & + & +- & -- & ++ & - & D \\
\hline & M-61 & + & + & - & - & ++ & + & - & - & + & + & + & - & - & + & - & - & - & + & - & - & ++ & -+ & + & -+ & +- & -+ & ++ & ++ & -+ & + & $-\quad-$ & -- & -+ & - & $\mathrm{D}$ \\
\hline & M-8 & $\mathrm{N}$ & + & - & + & ++ & + & + & - & + & + & + & $\mathbf{N}$ & $N$ & - & - & - & - & - & - & - & ++ & -+ & + & -+ & +- & -- & -+ & +- & + & + & $-\quad-$ & -- & -+ & - & $\mathrm{D}$ \\
\hline & M-99 & + & + & - & + & ++ & + & - & - & + & + & - & - & - & - & - & - & - & - & - & - & ++ & - & + & -+ & +- & -+ & -+ & +- & -+ & + & +- & -- & ++ & - & D \\
\hline & M- & - & + & - & + & ++ & + & + & - & + & + & + & + & - & - & - & - & - & - & - & - & ++ & + & + & - & -- & -- & -+ & +- & - & + & -- & -- & -+ & - & D \\
\hline & $\mathrm{M}-116^{\mathrm{T}}$ & + & + & - & + & ++ & & - & - & + & + & + & - & - & - & - & - & - & - & - & - & ++ & + & + & - & -- & -+ & -+ & +- & - & + & +- & -- & -+ & - & D \\
\hline 4 & M- & + & + & - & + & ++ & - & - & - & + & $T$ & + & - & - & 1 & + & . & - & - & - & - & ++ & -+ & + & -+ & ++ & + & ++ & ++ & -+ & + & ++ & $t+$ & -+ & - & DL \\
\hline & M-17 & + & + & - & + & ++ & + & - & - & + & + & + & - & - & + & + & + & - & - & - & - & ++ & + & + & -+ & ++ & + & ++ & ++ & -+ & + & ++ & $t+$ & -+ & - & $\mathrm{DL}$ \\
\hline & M-96 & + & + & - & + & ++ & + & - & - & + & + & + & - & - & + & + & + & - & - & - & - & ++ & -+ & + & + & $-\quad-$ & -+ & ++ & $+\quad+$ & - & + & ++ & - & ++ & - & DL \\
\hline & M-98 & + & + & - & + & ++ & - & - & - & + & + & - & - & - & + & - & - & - & _ & - & - & ++ & + & + & +- & -- & -+ & ++ & $+\quad+$ & + & + & $-\quad-$ & -- & ++ & - & $\mathrm{DL}$ \\
\hline 5 & $\mathrm{M}$ & + & + & - & - & ++ & + & + & - & + & $T$ & - & - & - & 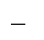 & - & 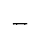 & . & - & - & - & ++ & + & + & - & - & -+ & -+ & ++ & - & +- & +- & -- & -+ & - & D \\
\hline & $\mathrm{M}-19^{\mathrm{T}}$ & + & + & - & - & ++ & + & - & - & + & + & + & + & - & - & - & - & - & - & - & - & ++ & + & + & - & -- & -+ & -+ & ++ & - & + & +- & -+ & ++ & - & D \\
\hline 6 & $\mathrm{X} 1-1$ & + & - & - & + & ++ & + & + & - & + & + & - & - & - & + & + & - & - & 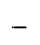 & - & - & ++ & + & + & - & -+ & + & ++ & ++ & - & + & +- & -- & -+ & - & $\mathrm{D}$ \\
\hline & X3-1 & + & + & - & - & ++ & + & + & - & + & - & - & - & - & + & + & + & - & + & + & + & ++ & + & + & ++ & ++ & + & ++ & $+\quad-$ & - & + & ++ & + & -+ & - & $\mathrm{D}$ \\
\hline & $\times 5-5$ & + & + & - & + & ++ & + & + & - & + & + & - & - & - & + & + & + & + & - & - & - & ++ & -+ & + & - & -+ & -+ & ++ & $+\quad+$ & - & + & ++ & + & +- & - & $\mathrm{D}$ \\
\hline & X6-1 & + & + & - & + & ++ & + & + & - & + & + & + & - & - & + & + & + & - & + & + & + & ++ & + & + & ++ & ++ & -+ & ++ & $+\quad+$ & + & + & +- & - & -+ & - & $\mathrm{D}$ \\
\hline & X9-1 & + & + & - & + & ++ & + & + & - & + & + & - & - & - & + & + & - & - & + & + & - & ++ & + & + & ++ & ++ & -+ & ++ & ++ & + & + & +- & -- & -+ & - & $\mathbf{N}$ \\
\hline & $\times 9-3$ & + & + & - & + & ++ & + & + & - & + & + & - & - & - & + & + & - & - & + & - & + & ++ & + & + & -+ & ++ & + & ++ & ++ & - & + & ++ & + & -+ & - & $\mathrm{D}$ \\
\hline & SH-3 & + & + & - & + & ++ & + & + & - & + & + & + & - & - & + & + & + & + & + & - & - & ++ & + & + & - & -+ & -+ & ++ & ++ & - & + & ++ & - & -+ & - & $\mathrm{D}$ \\
\hline & MB-1 & + & + & - & + & ++ & + & + & - & + & + & + & - & - & + & + & + & + & - & - & - & ++ & + & + & - & -+ & -+ & ++ & ++ & - & +- & ++ & + & ++ & - & $\mathrm{D}$ \\
\hline & MH-3 & + & + & - & + & ++ & + & + & - & + & + & - & - & - & - & + & - & - & + & - & + & ++ & -+ & + & ++ & ++ & -+ & ++ & ++ & + & + & ++ & + & -+ & - & D \\
\hline & I-3 & + & + & - & - & ++ & + & + & - & + & + & - & - & - & - & - & - & - & + & - & + & ++ & + & + & -+ & ++ & -- & + & -+ & + & + & ++ & + & ++ & - & $\mathrm{D}$ \\
\hline
\end{tabular}

${ }^{a}$ Data for groups 1 to 5 are from reference 33 .

${ }^{b}$ Type strain of $S$. inulinus.

${ }^{c}$ Data for the strains whose designations begin with $\mathrm{M}$ - are from reference 32

${ }^{d}+$, positive; - , negative; $\mathrm{N}$, not determined.

catalase-negative strains of Nakayama and Yanoshi are members of the genus Sporolactobacillus. Additional studies that include catalase-positive strains would make the taxonomic relationship between the genera Sporolactobacillus and Bacillus clearer.
Descriptions of the four new species and two new subspecies are given below. The following characteristics are common features of these species and subspecies of the genus Sporolactobacillus: gram-positive spore-forming rods that are generally 0.4 to 1.0 by 2.0 to $4.0 \mu \mathrm{m}$ in 7 -day-old cultures on GYP 
TABLE 2. Levels of DNA relatedness for isolates and some catalase-negative spore-forming lactic acid bacteria

\begin{tabular}{|c|c|c|c|c|c|c|c|c|}
\hline \multirow{2}{*}{$\begin{array}{l}\text { DNA homology } \\
\text { group }^{a}\end{array}$} & \multirow{2}{*}{$\begin{array}{l}\text { Source of } \\
\text { DNA }\end{array}$} & \multirow{2}{*}{$\begin{array}{c}\mathrm{G}+\mathrm{C} \text { content } \\
\quad(\mathrm{mol} \%)\end{array}$} & \multicolumn{6}{|c|}{ \% DNA-DNA reassociation with DNA from: } \\
\hline & & & $\mathrm{JCM} 6014^{\mathrm{T}}$ & $\mathrm{M}-114^{\mathrm{T}}$ & $\mathrm{M}-17^{\mathrm{T}}$ & $\mathrm{M}-116^{\mathrm{T}}$ & $\mathrm{M}-19^{\mathrm{T}}$ & $\mathrm{X} 1-1^{\mathrm{T}}$ \\
\hline \multirow[t]{2}{*}{1} & $\mathrm{JCM} 6014^{\mathrm{T}}$ & 47 & $100(100)^{b}$ & $25(22)$ & 24 & 22 & 22 & 12 \\
\hline & M-86 & 50 & 88 & 35 & 27 & & 19 & \\
\hline \multirow[t]{16}{*}{2} & M-45 & 45 & 32 & 75 & 47 & 38 & & \\
\hline & M-46 & 46 & 41 & 76 & & & & \\
\hline & M-50 & 45 & 41 & 81 & & & & \\
\hline & M-51 & 43 & 34 & 70 & & & & \\
\hline & M-54 & 45 & 42 & 72 & & & & \\
\hline & M-56 & 45 & 38 & 73 & & & & \\
\hline & M-59 & 45 & 33 & & 64 & 51 & & \\
\hline & M-84 & 46 & 47 & 101 & 60 & 52 & & \\
\hline & M-87 & 45 & 33 & 80 & & & 23 & \\
\hline & M-88 & 47 & $34(32)$ & $(77)$ & 61 & 35 & & 22 \\
\hline & M-101 & 47 & 41 & & 67 & 48 & & \\
\hline & M-103 & 47 & 33 & 78 & & & 21 & \\
\hline & M-113 & 45 & 43 & 86 & & & & \\
\hline & $\mathrm{M}-114^{\mathrm{T}}$ & 45 & $37(42)$ & $100(100)$ & 61 & 46 & & 28 \\
\hline & ME-1' & 47 & (30) & (82) & & & & 18 \\
\hline & F-4 & 47 & (34) & (73) & & & & 22 \\
\hline \multirow[t]{4}{*}{4} & M-13 & 46 & 22 & & 91 & 28 & & \\
\hline & $\mathrm{M}-17^{\mathrm{T}}$ & 45 & (36) & $(65)$ & 100 & 37 & & 37 \\
\hline & M-96 & 43 & 32 & 58 & & & & \\
\hline & M-98 & 45 & 26 & 57 & 84 & 41 & & \\
\hline \multirow[t]{8}{*}{3} & M-16 & 43 & 25 & & 40 & 55 & & \\
\hline & M-32 & 46 & 25 & & 43 & 64 & & \\
\hline & M-33 & 46 & 17 & & 30 & 64 & & \\
\hline & M-61 & 45 & 25 & & 46 & 72 & & \\
\hline & M-85 & 46 & 40 & 54 & 38 & 91 & & \\
\hline & M-99 & 45 & 35 & 48 & 41 & 63 & & \\
\hline & M-115 & 46 & 39 & & 38 & 119 & & \\
\hline & $\mathrm{M}-116^{\mathrm{T}}$ & 44 & 42 & 53 & 34 & 100 & & \\
\hline \multirow[t]{2}{*}{5} & M-18 & 43 & $24(26)$ & (24) & 29 & 19 & 101 & 32 \\
\hline & $\mathrm{M}-19^{\mathrm{T}}$ & 43 & 24 & 28 & 25 & 20 & 100 & \\
\hline \multirow[t]{11}{*}{6} & $\mathrm{X} 1-1^{\mathrm{T}}$ & 46 & (28) & (30) & & & & 100 \\
\hline & X3-1 & 45 & (25) & (27) & & & & 105 \\
\hline & X5-5 & 45 & (25) & (29) & & & & 104 \\
\hline & X6-1 & 46 & (25) & (32) & & & & 103 \\
\hline & X9-1 & 46 & (25) & (25) & & & & 98 \\
\hline & $X 9-3$ & 43 & (23) & (23) & & & & 86 \\
\hline & SH-3 & 46 & (25) & (33) & & & & 94 \\
\hline & MB-1 & 45 & (30) & (31) & & & & 76 \\
\hline & MH-3 & 44 & (23) & (24) & & & & 69 \\
\hline & I-3 & 46 & (22) & (23) & & & & 65 \\
\hline & Calf thymus & 43 & $0(4)$ & $1(4)$ & 4 & 5 & 1 & 2 \\
\hline
\end{tabular}

${ }^{a}$ All of the homology groups except group 6 correspond to the groups described by Yanagida et al. (33).

${ }^{b}$ The values in parentheses are from reference 33 .

agar; generally motile; catalase and cytochromes are absent; facultatively anaerobic; good growth occurs on media containing glucose, and poor or no growth occurs in nutrient broth; Dor DL-lactic acid is produced homofermentatively; acid is produced from glucose, fructose, and mannose; the cellular fatty acid profile includes branched-chain acids, and anteiso- $\mathrm{C}_{15: 0}$ and anteiso- $\mathrm{C}_{17: 0}$ are the predominant fatty acids; the cell wall contains meso-diaminopimelic acid; and the major isoprenoid quinone is MK-7. The type strains of the taxa described below have been deposited in the Japan Collection of Microorganisms, The Institute of Physical and Chemical Research (RIKEN), Wako-shi, Saitama, Japan.
Description of Sporolactobacillus nakayamae subsp. nakayamae sp. nov., subsp. nov. Sporolactobacillus nakayamae subsp. nakayamae (na.ka.ya'mae. M.L. gen. n. nakayamae, of Nakayama, named for Ooki Nakayama, a Japanese microbiologist who isolated a number of Sporolactobacillus strains). Most strains grow at $15^{\circ} \mathrm{C}$. Acid is produced from maltose, but not from arabinose, ribose, xylose, rhamnose, and lactose. Most strains produce acid from galactose, sucrose, and trehalose. Most strains do not produce acid from cellobiose, melibiose, salicin, and starch. The DNA $\mathrm{G}+\mathrm{C}$ content ranges from 43 to $47 \mathrm{~mol} \%$ (as determined by the $T_{m}$ method). The habitat is soil. The type strain is strain M-114 (= JCM 3514). The 
TABLE 3. Salient characteristics of the genera of aerobic, endospore-forming bacteria

\begin{tabular}{|c|c|c|c|c|c|c|c|c|c|}
\hline Genus & $\begin{array}{l}\text { Rod- } \\
\text { shaped } \\
\text { cells }\end{array}$ & $\begin{array}{l}\text { Spore } \\
\text { shape }\end{array}$ & $\begin{array}{l}\text { Anaerobic } \\
\text { growth }\end{array}$ & $\begin{array}{l}\text { Growth in } \\
\text { nutrient } \\
\text { broth }\end{array}$ & $\begin{array}{l}\text { Catalase } \\
\text { activity }\end{array}$ & $\begin{array}{l}\text { Production } \\
\text { of lactic } \\
\text { acid }\end{array}$ & $\begin{array}{l}\text { Major } \\
\text { isoprenoid } \\
\text { quinone }\end{array}$ & $\begin{array}{l}\text { Major cellular } \\
\text { fatty acids }\end{array}$ & $\begin{array}{l}\mathrm{G}+\mathrm{C} \\
\text { content } \\
\text { (mol\%) }\end{array}$ \\
\hline Sporolactobacillus ${ }^{a}$ & $t^{b}$ & Oval & + & - & - & + & MK- $7^{c}$ & anteiso- $\mathrm{C}_{15: 0}$, anteiso- $\mathrm{C}_{17: 0}$ & $43-50$ \\
\hline Amphibacillus & + & Oval & + & - & - & + & None & $\begin{array}{l}\text { anteiso- } C_{15: 0}, C_{16: 0} \text {, iso- } C_{16: 0} \\
\quad \text { iso- } C_{15: 0}\end{array}$ & $36-38$ \\
\hline Bacillus $^{e}$ & + & $\begin{array}{l}\text { Oval or } \\
\text { spherical }\end{array}$ & V & + & + & V & MK-7 & $\mathrm{V}$ & $32-69$ \\
\hline Brevibacillus ${ }^{f}$ & + & Oval & V & + & + & NT & MK-7 & $\begin{array}{l}\text { anteiso- } C_{15: 0} \text { and iso- } C_{15: 0} \text { or } \\
\text { iso- } C_{15: 0}\end{array}$ & $46-57$ \\
\hline Paenibacillus ${ }^{g}$ & + & Oval & + & + & + & NT & MK-7 & anteiso- $\mathrm{C}_{15: 0}$ & $40-54$ \\
\hline Sporosarcina ${ }^{h}$ & - & Round & - & + or - & + & NT & MK-7 & & $40-42$ \\
\hline
\end{tabular}

${ }^{a}$ Data from reference 12 and this study.

${ }^{b}+$, positive; - , negative; $V$, variable; NT, not tested.

${ }^{c}$ MK-7, menaquinone 7 .

${ }^{d}$ Data from reference 20

${ }^{e}$ Data from reference 3

${ }^{f}$ Data from references 23 and 28 .

${ }^{g}$ Data from references 8 and 9 .

${ }^{h}$ Data from reference 4 .

other strains examined are strains M-45, M-46, M-47, M-50, M-51, M-54, M-56, M-59, M-84, M-87, M-88, M-101, M-103, $\mathrm{M}-113, \mathrm{ME}-1$ ', and F-4.

Description of Sporolactobacillus nakayamae subsp. racemicus subsp. nov. Sporolactobacillus nakayamae subsp. racemicus (ra.ce.mi'cus. L. n. racemus, rotatory of lactic acid racemi, [rotatory]-lactic acid). DL-Lactic acid is produced. Litmus milk is acidified. Acid is produced from galactose, maltose, melibiose, sucrose, raffinose, trehalose, and inulin, but not from arabinose, ribose, and xylose. The DNA G $+\mathrm{C}$ content ranges from 43 to $46 \mathrm{~mol} \%$ (as determined by the $T_{m}$ method). The habitat is soil. The type strain is strain M-17 (= JCM 3417). The other strains examined are strains $M-13, M-96$, and M-98.

Description of Sporolactobacillus terrae sp. nov. Sporolactobacillus terrae (ter'rae. M.L. gen. n. terrae, of the earth). Most strains grow at $15^{\circ} \mathrm{C}$. Acid is produced from trehalose and inulin, but not from ribose, xylose, rhamnose, lactose, and sorbitol. Most strains produce acid from galactose and sucrose. Most strains do not produce acid from arabinose, melibiose, and starch. The DNA G+C content ranges from 43 to 46 mol\% (as determined by the $T_{m}$ method). The habitat is soil. The type strain is strain M-116 (= JCM 3516). The other strains examined are strains M-16, M-32, M-33, M-61, M-85, $\mathrm{M}-99$, and M-115.

Description of Sporolactobacillus kofuensis sp. nov. Sporolactobacillus kofuensis (ko.fu.en'sis. M.L. adj. kofuensis, belonging to Kofu, Yamanashi, Japan, the source of the soil from which the organism was isolated). Acid is produced from galactose, maltose, sucrose, raffinose, trehalose, mannitol, and inulin, but not from arabinose, ribose, xylose, rhamnose, cellobiose, lactose, melibiose, salicin, and sorbitol. The DNA G $+\mathrm{C}$ content is $43 \mathrm{~mol} \%$ (as determined by the $T_{m}$ method). The habitat is soil. The type strain is strain M-19 (= JCM 3419). The other strain examined is strain $\mathrm{M}-18$.

Description of Sporolactobacillus lactosus sp. nov. Sporolactobacillus lactosus (lac.to'sus. M.L. adj. lactosus, of lactose, pertaining to lactose). Litmus milk is acidified and then reduced by some strains. Acid is produced from galactose, lactose, melibiose, and trehalose. Most strains produce acid from maltose, sucrose, raffinose, and inulin. The DNA G+C content ranges from 43 to $46 \mathrm{~mol} \%$ (as determined by the $T_{m}$ method) The habitat is soil in Japan and fermentation starters. The type strain is strain X1-1 (= JCM 9690). The other strains examined are strains X3-1, X5-5, X6-1, X9-1, X9-3, SH-3, MB-1, MH-3, and I-3.

TABLE 4. Differential characteristics of Sporolactobacillus species and subspecies

\begin{tabular}{|c|c|c|c|c|c|c|c|c|c|c|c|c|c|}
\hline \multirow[b]{2}{*}{ Species or subspecies } & \multirow{2}{*}{ 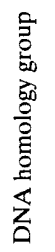 } & \multirow[b]{2}{*}{ 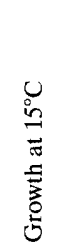 } & \multicolumn{2}{|c|}{ Litmus milk } & \multicolumn{8}{|c|}{ Acid production from carbohydrates } & \multirow{2}{*}{ 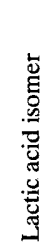 } \\
\hline & & & 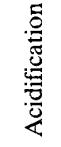 & 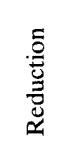 & 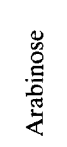 & $\frac{\mathscr{u}}{\stackrel{x}{x}}$ & 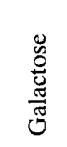 & 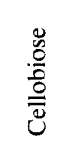 & 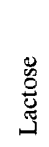 & 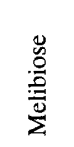 & $\begin{array}{l}\bar{c} \\
\bar{y} \\
\bar{n}\end{array}$ & 鸹 & \\
\hline S. inulinus & 1 & $-{ }^{a}$ & - & - & - & - & - & - & - & - & + & $\mathrm{d}$ & $\mathrm{D}$ \\
\hline S. nakayamae subsp. nakayamae & 2 & $(+)$ & d & $(-)$ & - & - & $(+)$ & $(-)$ & - & $(-)$ & $(-)$ & $(-)$ & $\mathrm{D}$ \\
\hline S. nakayamae subsp. racemicus & 4 & + & + & $\mathrm{d}$ & - & - & + & $\mathrm{d}$ & $\mathrm{d}$ & + & $\mathrm{d}$ & + & $\mathrm{DL}$ \\
\hline S. terrae & 3 & $(+)$ & $(-)$ & - & $(-)$ & - & $(+)$ & d & - & $(-)$ & $(-)$ & + & $\mathrm{D}$ \\
\hline S. kofuensis & 5 & - & - & - & - & - & + & - & - & - & $d$ & + & $\mathrm{D}$ \\
\hline S. lactosus & 6 & $(+)$ & $(+)$ & $(+)$ & d & $\mathrm{d}$ & + & d & + & + & d & $(+)$ & $\mathrm{D}$ \\
\hline
\end{tabular}

${ }^{a}-$, all strains are negative; + , all strains are positive; $(-)$, at least $80 \%$ of the strains are negative; $(+)$, at least $80 \%$ of the strains are positive; $\mathrm{d}, 20$ to $80 \%$ of the strains are positive. 


\section{ACKNOWLEDGMENTS}

We thank the late O. Nakayama, Yamanashi University, Kofu, Japan, for kindly supplying cultures and also for his valuable comments. We thank Setsu Hitokoto and Norifumi Shimono for technical assistance.

\section{REFERENCES}

1. Andersch, I., S. Pianka, D. Fritze, and D. Claus. 1994. Description of Bacillus laevolacticus (ex Nakayama and Yanoshi 1967) sp. nov., nom. rev. Int. J. Syst. Bacteriol. 44:659-664.

2. Ash, C., J. A. E. Farrow, S. Wallbanks, and M. D. Collins. 1991. Phylogenetic heterogeneity of the genus Bacillus revealed by comparative analysis of small-subunit-ribosomal RNA sequences. Lett. Appl. Microbiol. 13:202-206.

3. Claus, D, and R. C. W. Berkeley. 1986. Genus Bacillus Cohn 1872, p. 1105-1140. In P. H. A. Sneath, N. S. Mair, M. E. Sharpe, and J. G. Holt (ed.) Bergey's manual of systematic bacteriology, vol. 2. The Williams \& Wilkins Co., Baltimore, Md.

4. Claus, D., and F. Fahmy. 1986. Genus Sporosarcina Kluyver and van Niel 1936, p. 1202-1206. In P. H. A. Sneath, N. S. Mair, M. E. Sharpe, and J. G. Holt (ed.), Bergey's manual of systematic bacteriology, vol. 2. The Williams \& Wilkins Co., Baltimore, Md.

5. Collins, M. D., and D. Jones. 1979. Isoprenoid quinone composition as a guide to the classification of Sporolactobacillus and possibly related bacteria. J. Appl. Bacteriol. 47:293-297.

6. Doores, S., and D. C. Westhoff. 1983. Selective method for isolation of Sporolactobacillus from food and environmental sources. J. Appl. Bacteriol. 54:273-280.

7. Fox, G. E., K. R. Pechman, and G. R. Woese. 1977. Comparative cataloging of $16 \mathrm{~S}$ ribosomal ribonucleic acid: molecular approach to procaryotic systematics. Int. J. Syst. Bacteriol. 27:44-57.

8. Heyndrickx, M., K. Vandemeulebroecke, B. Hoste, P. Janssen, K. Kersters, P. De Vos, N. A. Logan, N. Ali, and R. C. W. Berkeley. 1996. Reclassification of Paenibacillus (formerly Bacillus) pulvifaciens (Nakamura 1984) Ash et al. 1994, a later subjective synonym of Paenibacillus (formerly Bacillus) larvae (White 1906) Ash et al. 1994, as a subspecies of P. larvae, with emended description of $P$. larvae as $P$. larvae subsp. larvae and $P$. larvae subsp. pulvifaciens. Int. J. Syst. Bacteriol. 46:270-279.

9. Heyndrickx, M., K. Vandemeulebroecke, P. Scheldeman, K. Kersters, P. D Vos, N. A. Logan, A. M. Aziz, N. Ali, and R. C. W. Berkeley. 1996. A polyphasic reassessment of the genus Paenibacillus, reclassification of Bacillus validus (Nakamura 1984) as Paenibacillus lautus comb. nov. and of $B a-$ cillus peoriae (Montefusco et al. 1993) as Paenibacillus peoriae comb. nov. and emended descriptions of $P$. lautus and of $P$. peoriae. Int. J. Syst. Bacteriol. 46:988-1003.

10. Ikemoto, S. H. Kuraishi, K. Komagata, R. Azuma, T. Suto, and H. Murooka 1978. Cellular fatty acid composition in Pseudomonas species. J. Gen. Appl. Microbiol. 24:119-213.

11. Johnson, J. L. 1984. Nucleic acids in bacterial classification, p. 8-11. In N. R Krieg and J. G. Holt (ed.), Bergey's manual of systematic bacteriology, vol 1. The Williams \& Wilkins Co., Baltimore, Md.

12. Kandler, O, and N. Weiss. 1986. Genus Sporolactobacillus Kitahara and Suzuki 1963 , $69^{\mathrm{AL}}$, p. 1139-1141. In P. H. A. Sneath, N. S. Mair, M. E. Sharpe, and J. G. Holt (ed.), Bergey's manual of systematic bacteriology, vol 2. The Williams \& Wilkins Co., Baltimore, Md.

13. Kitahara, K., and C. L. Lai. 1967. On the spore formation of Sporolactoba cillus inulinus. J. Gen. Appl. Microbiol. 13:197-203.
14. Kitahara, K., and J. Suzuki. 1963. Sporolactobacillus nov. subgen. J. Gen. Appl. Microbiol. 9:59-71.

15. Komagata, K., and K. Suzuki. 1987. Lipid and cell-wall analysis in bacterial systematics. Methods Microbiol. 19:161-207.

16. Marmur, J., and P. Doty. 1962. Determination of the base composition of deoxyribonucleic acid from its thermal denaturation temperature. J. Mol. Biol. 5:109-118.

17. Mitsuoka, T. 1969. Vergleichende Untersuchungen uber die Lactobazillenaus den Faeces von Menschen, Schweinen und Huhnern. Zentralbl. Bakteriol. Parasitenkd. Infektionskr. Hyg. Abt. 1 Orig. 210:32-51.

18. Nakayama, O., and M. Yanoshi. 1967. Spore-bearing lactic acid bacteria isolated from rhizosphere. I. Taxonomic studies on Bacillus laevolacticus nov. sp. and Bacillus racemilacticus nov. sp. J. Gen. Appl. Microbiol. 13:139-153.

19. Nakayama, O., and M. Yanoshi. 1967. Spore-bearing lactic acid bacteria isolated from rhizosphere. II. Taxonomic studies on the catalase-negative strains. J. Gen. Appl. Microbiol. 13:155-165.

20. Niimura, Y, E, Koh, F. Yanagida, K. Suzuki, K. Komagata, and M. Kozaki. 1990. Amphibacillus xylanus gen. nov., sp. nov., a facultatively anaerobic sporeforming xylan-digesting bacterium which lacks cytochrome, quinone, and catalase. Int. J. Syst. Bacteriol. 40:297-301.

21. Okada, S., T. Toyoda, and M. Kozaki. 1978. An easy method for the determination of the optical types of lactic acid produced by lactic acid bacteria. Agric. Biol. Chem. 42:1781-1783.

22. Saito, H., and K. Miura. 1963. Preparation of transforming deoxyribonucleic acid by phenol treatment. Biochim. Biophys. Acta 72:619-629.

23. Shida, O., H. Takagi, K. Kadowaki, L. K. Nakamura, and K. Komagata. 1995. Proposal of Bacillus reuszeri sp. nov., Bacillus formosus sp. nov., nom. rev., and Bacillus borstelensis sp. nov., nom. rev. Int. J. Syst. Bacteriol. $\mathbf{4 5}$ 93-100.

24. Skerman, V. B. D., V. McGovan, and P. H. A. Sneath (ed.). 1980. Approved lists of bacterial names. Int. J. Syst. Bacteriol. 30:225-420.

25. Staneck, J. L., and G. D. Roberts. 1974. Simplified approach to identification of aerobic actinomycetes by thin-layer chromatography. Appl. Microbiol 28:226-237.

26. Suzuki, K., T. Kaneko, and K. Komagata. 1981. Deoxyribonucleic acid homologies among coryneform bacteria. Int. J. Syst. Bacteriol. 31:131-138.

27. Suzuki, T., and K. Yamasato. 1994. Phylogeny of spore-forming lactic acid bacteria based on 16S rRNA gene sequences. FEMS Microbiol. Lett. 115: 13-18.

28. Takagi, H., O. Shida, K. Kadowaki, K. Komagata, and S. Udaka. 1993. Characterization of Bacillus brevis, with descriptions of Bacillus migulanus $\mathrm{sp}$ nov., Bacillus chosinensis sp. nov., Bacillus parabrevis sp. nov., and Bacillus galactophilus sp. nov. Int. J. Syst. Bacteriol. 43:221-231.

29. Tamaoka, J., Y. Katayama-Fujimura, and H. Kuraishi. 1983. Analysis of bacterial menaquinone mixtures by high-performance liquid chromatography. J. Appl. Bacteriol. 54:31-36.

30. Uchida, K., and K. Mogi. 1973. Cellular fatty acid spectra of Sporolactobacillus and some other Bacillus-Lactobacillus intermediates as a guide to their taxonomy. J. Gen. Appl. Microbiol. 19:129-140.

31. Weiss, N., R. Plapp, and O. Kandler. 1967. Die Aminosauresequenz desDAP-haltigen Mureins von Lactobacillus plantarum und Lactobacillus inulinus. Arch. Mikrobiol. 58:313-323.

32. Yanagida, F, K. Suzuki, T. Kaneko, M. Kozaki, and K. Komagata. 1987. Morphological, biochemical, and physiological characteristics of spore-forming lactic acid bacteria. J. Gen. Appl. Microbiol. 33:33-45.

33. Yanagida, F., K. Suzuki, T. Kaneko, M. Kozaki, and K. Komagata. 1987 Deoxyribonucleic acid relatedness among some spore-forming lactic acid bacteria. J. Gen. Appl. Microbiol, 33:47-55. 